\title{
Relative validity of a food frequency questionnaire to assess fruit and vegetable intake in healthy older adults
}

\section{Abstract}

Accurate assessment of dietary intake in older populations is important for determining the role of diet in healthy ageing. The food frequency questionnaire (FFQ) is a commonly used dietary assessment tool, however there is limited evidence regarding its utility for accurately assessing fruit and vegetable (FV) intake in older adults. The objective of this study was to validate FV intakes estimated from the FFQ used in the Northern Ireland Cohort for the Longitudinal Study of Ageing (NICOLA) against a food diary (FD).

A dietary validation study was conducted in a sub-sample of 95 participants (45 males, 50 females, aged $>50$ years) from NICOLA. Participants were asked to complete a FFQ and 4-day FD (reference method) at two time-points (Month 0 and Month 6). Self-reported FV intakes were compared between methods using Spearman's correlation coefficients, examining the percentage of participants classified into the same or adjacent quartile of FV intake, weighted kappa and Bland-Altman plots.

Median fruit, vegetable and total FV intake were significantly higher in the FFQ than the FD at both Month 0 and Month 6 (all $p<0.001$ ). Significant positive correlations (all $p<0.05$ ) were observed between the FFQ and FD estimates of FV intake at both timepoints (Month 0, $\mathrm{r}=0.57,0.50$ and 0.49 for fruit, vegetables, total $\mathrm{FV}$, respectively; Month $6 \mathrm{r}=0.56,0.42$ and 0.50 , respectively). When FV intakes were classified into fourths (based upon quartiles of total FV portions by FD or FFQ), $80 \%$ and $79 \%$ of participants were classified into the same or adjacent quartile at Month 0 and Month 6, respectively. Weighted kappa indicated a fairmoderate agreement between the two methods for FV intake (weighted kappa $=0.35$ and 0.37 at Month 0 and Month 6, respectively). Bland-Altman plots showed that, as FV intake increased, there was a widening in limits of agreements, between the FFQ and FD. There was also a significant positive correlation noted between total FV intakes reported at Month 0 and those reported at Month 6 $(\mathrm{r}=0.70, \mathrm{p}<0.001)$.

Over-reporting of FV intake was evident with the FFQ compared to the FD, however, the results showed good comparability between the methods in being able to rank older adults according to their FV intake. An additional analysis of FV biomarkers obtained from this sample will provide a more objective assessment of FV intake by each method.

\section{Conflict of Interest}

There is no conflict of interest 